\title{
Investigation of Lightning Direct Effects on Aircraft Materials
}

\author{
D.Mitchard*, N S Jamoshid, D. Clark, D. Carr, A. Haddad \\ Morgan-Botti Lightning Laboratory, Advanced High Voltage Engineering Research Centre, \\ School of Engineering, Cardiff University, Cardiff, UK \\ *mitcharddr@cf.ac.uk
}

\begin{abstract}
Lightning is one of the most unpredictable and destructive forces in nature, and ensuring the protection and safety of aircraft in flight, as well as other modes of airborne transport, when struck by lightning presents a unique set of engineering challenges. In order to progress into a new generation of conductive carbon composites and/or embedded light-weight lightning protection systems, further scientific understanding is required on the mechanisms and impact of lightning on carbon composite aircraft. This paper recalls how modern lightning research has progressed from deducing information from natural lightning events to reproducible and instrumented lightning generators capable of accurate and repeatable experimentation. Results of investigations on how aerospace materials, with and without lightning protection, react when struck by lightning are presented illustrating the extent of damage that could be caused. Furthermore, methods of studying lightning effects, such as mechanical deflection, chemical element interaction, and temperature measurements, are then presented to illustrate the role of lightning experimentation as a means to help support aspects of modelling such as material behavior, aircraft design and potentially flight performance.
\end{abstract}

Keywords - Lightning, aircraft, carbon composite, materials, damage, experiment, modelling

\section{INTRODUCTION}

Lightning is one of the most destructive forces in nature. It is characterized by an average current of 30,000 Amperes and electric potential of several million kilovolts delivered within $100 \mu \mathrm{s}$ to an impact area less than a square centimeter, resulting in temperatures in the region of $30,000 \mathrm{~K}$, pressures of tens of $\mathrm{MPa}$ and a powerful electromagnetic pulse capable of disrupting, and sometimes destroying, electric and electronic systems. It is an inherently random event and, although the chance of a lightning strike can be significantly reduced, it can never be entirely eliminated. Most structures and modes of transport are design to withstand lightning strikes with minimal adverse effects by using a variety of methods that typically rely on creating a clear path to ground, safely diverting the lightning current path. However, aircraft and other airborne transport present a unique set of engineering challenges as a unique path to ground cannot be established while in flight; this is further complicated by such transport occasionally initiating lightning strikes as they can introduce a conductive link between, for example, a cloud and the Earth. Statistically, each commercial airliner experiences a lightning strike, on average, once per year. Although much is known about the lightning mechanisms

This work was sponsored by Sêr Cymru National Research Network in Advanced Engineering and Materials. and characteristics, a number of recent discoveries have highlighted previously unknown phenomena [1] and a general increase in worldwide lightning activity has been observed which some have linked to climate change [2].

The advent of new composite materials, such as carbon composites used in the skin and sub-structures of many new commercial airliners, has added another dimension to this as, unlike traditional conductive metals, they often behave in complex and sometimes destructive ways when struck by lightning. Carbon composites offer many advantages over traditional metals as they are lighter, stiffer and experience minimal corrosion but, unlike highly conductive metals, they are generally non-conducting and thus cannot efficiently dissipate lightning energy. This can result in complex and sometimes destructive behavior which is mitigated through lightning protection systems that reintroduce metallic meshes into an aircraft structure countering weight-saving initiatives. In order to progress into a new generation of conductive carbon composites and/or embedded light-weight lightning protection systems, further scientific understanding is required on the mechanisms and impact of lightning on carbon composite aircraft. This will be achieved by combined experimentation, computation and analytical studies that will feed into key modelling aspects such as material properties and component design, supporting safer, secure and improved flight performance.

\section{Lightning TeSt FACILITIES}

The need to understand how lightning interacts with modern composites, particularly in the aerospace sector, has led to the creation of commercial research direct-effect lightning facilities, including the unique university-based research facility, the Morgan-Botti Lightning Laboratory (MBLL) at Cardiff University [3] dedicated to lightning and aerospace research. A typical lightning test generator is designed to replicate the most destructive aspects of a lightning strike, namely the delivery of a large current within a short period, with voltages often practically limited to tens of $\mathrm{kV}$ rather than GV as seen in natural lightning. Furthermore, many generators are capable of producing worst-case lightning strike scenarios up to $200 \mathrm{kA}$. The complete set of aerospace lightning waveforms and combinations thereof are specified in the aerospace standard EUROCAE ED84 [4]. In this paper, only results from the most common waveform are described, a $100 \mathrm{kA}$ peak with an 18/40 shape that is a critically damped oscillatory waveform generated by the MBLL A/D bank [3]. 
Laboratory experiments to investigate the impact of lightning on an aerospace material typically involve securing a $50 \times 50 \mathrm{~cm}$ material sample to a fixed test rig. A lightning electrode is positioned several centimeters above the material and one end of a $0.1 \mathrm{~mm}$ diameter metallic initiation wire is tied to the electrode while the other end is secured to the material surface with tape; the wire is used to guide the lightning arc to a specific impact point and will typically vaporize within a few microseconds of the arc forming. Further details are given in [3] and [4]. Adequately shielded and protected diagnostic equipment, such as voltage and current recording equipment, still and high-speed cameras, temperature and pressure probes are frequently used to capture data from the application of a lightning strike to the test object. Figure 1 shows a test setup when a $100 \mathrm{kA}$ lightning current is applied to a carbon composite panel. Other diagnostics can also be used before and after the tests, e.g. electrical and mechanical characterizations, sectioning and microscopy, and nondestructive techniques such as ultrasonic and x-ray scans to further understand the impact of the lightning strike on the material being studied.

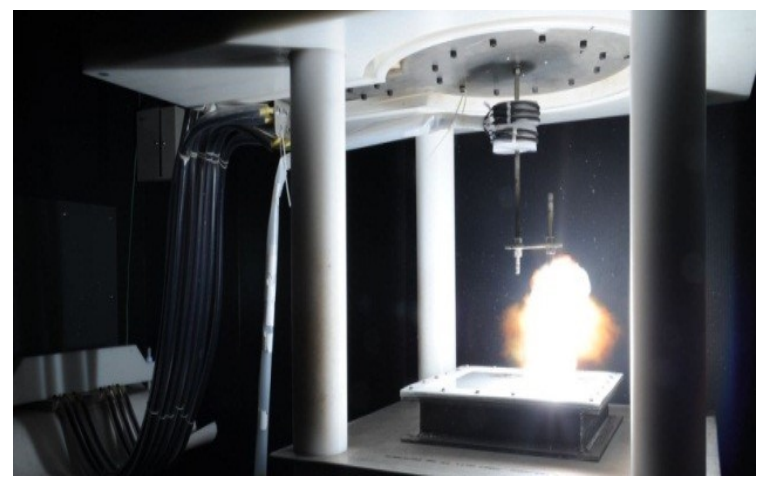

Fig. 1: An example of a $100 \mathrm{kA}$ generated lightning strike at MBLL. The image was taken using a shielded and filtered digital still camera.

\section{LIGHTNING DAMAGE}

Although the introduction of carbon composite materials into the aerospace industry had many advantages, such as being lighter, stiffer and less prone to corrosion, an outstanding shortfall is their inherently less-conductive nature in contrast to traditional highly conductive metals. As much of the composite is used in the skin and sub-structure of an aircraft, a large amount of metallic mesh is reintroduced into the airframe in order to provide sufficient lightning protection. This is counter to the weight-saving and minimal corrosion advantages of carbon composites lowering overall aircraft efficiency and increasing inspection and maintenance times. However, this is not just limited to the aerospace industry as the introduction of non-conductive composites into other industry sectors, such as wind turbine structures, power infrastructure, shipping and rail electrification, will present similar problems.

The ability to design materials to effectively dissipate and/or transform lightning energy into other forms, such as light, heat and physical movement, with minimal adverse effects is critical to the safety of their structural integrity and for the protection of electrical, electronic and other critical systems contained within their structure. A direct lightning strike can result in physical damage to a material surface, internal structure and joints, leading to burning, melting, vaporization and punctures. Surface explosions and electromagnetic and electromechanical forces are dominant contributors to large-scale effects such as mechanical displacement, deflection, vibration and delamination of composites, with cases of extreme destruction at the point of injection [5-8].

To illustrate the difference in the level of damage caused, three samples were chosen to represent traditional aerospace metal (aluminum), unprotected composite (carbon fiber) and protected composite frequently used in modern aircraft (carbon fiber with copper mesh). Each sample was photographed, subjected to a $100 \mathrm{kA}$ lightning current of D-type waveform at MBLL, then photographed again in order to assess the level of damage. The results are illustrated in Figure 2.
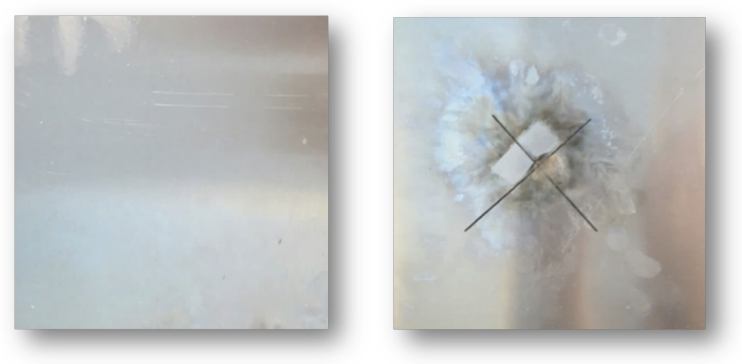

(a) Aluminium sample before and after the strike
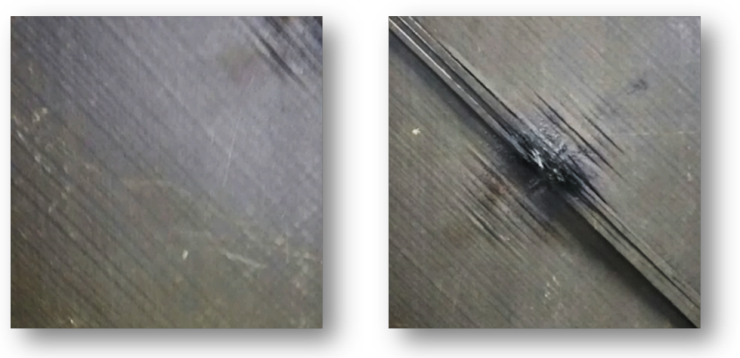

(b) Carbon fibre composite sample before and after the strike
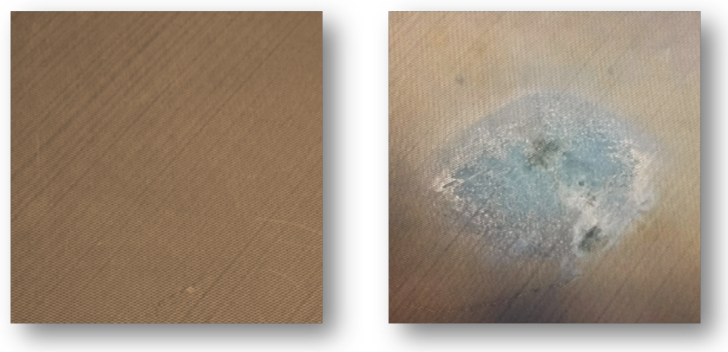

(c) Carbon fibre composite with copper mesh before and after the strike

Fig. 2: Examples of lightning strike damage to three different types material representing aircraft skin, showing before (left) and after (right) the strike. Each image is $10 \times 10 \mathrm{~cm}$. The rectangular undamaged area in (a) was due to the presence of tape securing the initiation wire. 
As can be seen from Figure 2(c), the introduction of a surface copper mesh onto a carbon composite material surface offered a satisfactory level of protection close to that of aluminum shown in Fig. 2(a). In both cases, the damage was largely restricted to the surface as the conductive metal was able to efficiently dissipate the lightning energy causing minimal damage and retaining structural integrity of the material as a whole. In the case of Fig. 2(c), although some of the mesh had vaporized and some surface burns are present, the underlying carbon composite is hardly damaged. On an aircraft, this area would subsequently be assessed and patched as required. In addition, like the aluminum case, the copper mesh also offers a level of shielding to underlying electric and electronic devices. In contrast, the unprotected carbon composite sample, shown in Fig. 2(b), clearly suffered damage over a large area where several layers of delaminated fibers are evident. In the figure, it can be seen that many of the fibers were broken and some surface fibers up to $25 \mathrm{~cm}$ long have become entirely detached. Further, localized burnt fibers and resin are clearly visible, with much of the resin being vaporized at the current attachment point and a sizeable impact crater 8 $\mathrm{mm}$ in diameter and $3 \mathrm{~mm}$ deep is present; this can compromise the structural integrity of the material as a whole. Without the protective metallic mesh, the carbon composite material would offer no protection to underlying electrical or electronic devices.

\section{Mechanical Defelection Under Lightning StRikeS}

In order to further understand and characterise lightning and its direct effects on materials, a number of tests and modelling techniques have been developed and used at MBLL, contributing towards the design and application of carbon composite components. Well established experimental techniques have now been developed to characterise the electrical behaviour of carbon composites materials, such as conductivity and associated distribution of current flow, and appropriate models with a good level of accuracy have been derived. However, electromechanical behaviour is less well understood and is dependent on material properties such as Young's modulus, Poisson's Ratio and density leading to its ability to withstand large physical forces, such as the Lorentz forces and supersonic pressure shockwaves which exist during a lightning strike event [8]. This is a more complex consideration for carbon composites, as such properties can vary depending on the direction of the material fibres; for example, a multi-layer composite has very different throughthickness properties to its planar properties. Nevertheless, a number of experiments to study how composites physically react to a lightning strike have been carried out [5-8].

To explore the mechanical response of a material subjected to a lightning strike, a $2 \mathrm{~mm}$ thick $50 \times 50 \mathrm{~cm}$ aluminium plate was tested in the MBLL test rig. The centre of the topside of the plate was struck with a $100 \mathrm{kA} 100 \mu$ s lightning waveform while the underside was filmed using a 200,000 fps high-speed camera. A drawn-on grid and floodlights were used to enhance the visualisation of material deformation. An example of three images from the camera are given in Figure 3. Figure 3 (a) shows the plate to be completely flat before the strike, whereas Figure 3 (b) shows the formation of a radial deformed area 100 $\mu \mathrm{s}$ after triggering the lightning strike. The deformed area is seen to be much larger $200 \mu$ s after triggering the strike and, in fact, continues to grow until around $400 \mu \mathrm{s}$, at which point the material begins to rebound. The relatively slow material response in comparison to the quicker lightning current shape is attributed to material inertia. Beyond $400 \mu \mathrm{s}$, the material experienced a level of damped oscillation that is typical for almost all such materials.

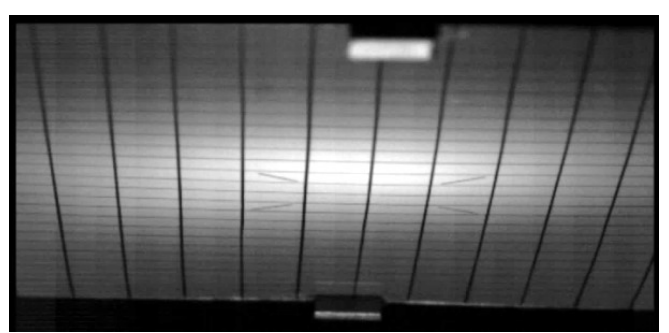

(a) Before the lightning strike



(b) $100 \mu \mathrm{s}$ after triggering the lightning strike

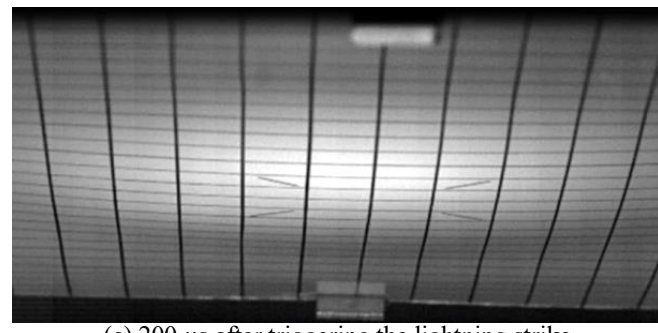

(c) $200 \mu$ s after triggering the lightning strike

Fig. 3: Images taken with a high-speed camera from the underside of a $2 \mathrm{~mm}$ aluminium plate before and after being struck with a $100 \mathrm{kA}$ lightning current waveform.

The mechanical distortion of the aluminium sample under a lightning current waveform was compared with the response of the same material under a direct mechanical impact using a calibrated $5 \mathrm{~J}$ drop-weight. In both cases, the movement of the central impact point was measured using a technique developed to track the position of the grid point on each individual frame of the high-speed camera footage [10]. Using this approach, the measured deflection of the centre point was plotted and is presented in Figure 4. It can be seen from Figure 4(a), obtained when a $100 \mathrm{kA}$ lightning current waveform is injected into the aluminium sample, that the lightning impact was translated into a relatively large oscillation, with only a small level of initial deflection. It also rebounds further than it deflects, which is thought to be a result of air pressure differences resulting from the supersonic shockwave on the surface which accompanies 
the lightning arc [10]. Figure 4(b), on the other hand, shows a much larger initial deflection mainly because the mechanical impact head carried its momentum through and stayed in contact with the plate for $18 \mathrm{~ms}$ (this is compared to $100 \mu \mathrm{s}$ for a lightning strike), resulting in a less energetic oscillation phase particularly in the initial phases. The high noise level that can be seen in both results of Figure 4 are attributed to deflection and oscillation waves which have been reflected from the constrained edges of the aluminum plate back to the measured center measurement point resulting in constructive and destructive interference.

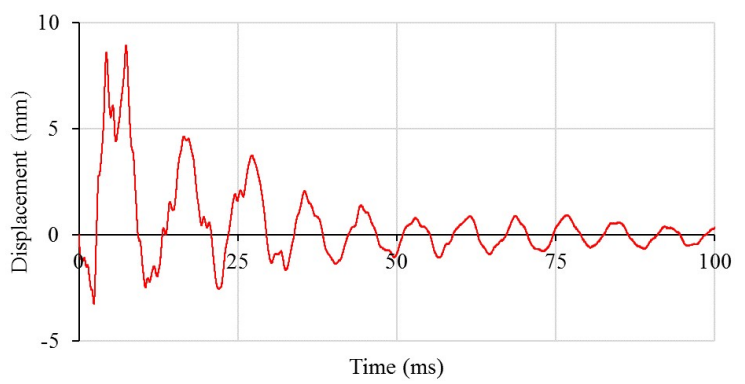

(a) $100 \mathrm{kA}$ lightning strike to $2 \mathrm{~mm}$ aluminium

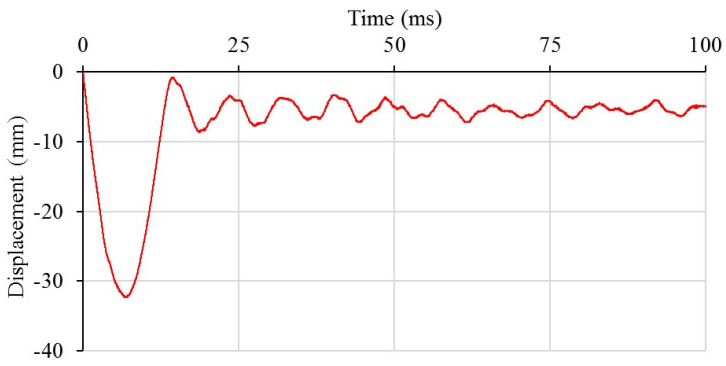

(b) $5 \mathrm{~J}$ drop-weight test on $2 \mathrm{~mm}$ aluminium

Fig. 4: Deflection and oscillation of aluminum plates after a lightning strike and drop-weight test.

It is generally accepted that, in both cases exemplified in figure 4, virtually all of the damage to a material is caused before the oscillation phase. This is because the material is unable to react to the large amount of energy delivered within the limitations of its own physical properties resulting in the force exceeding yield strength which, in turn, causes permanent damage; this in the form of a dent for a metal like aluminum and cracks and delamination for a composite such as carbon composite. In the case of carbon composites, it is postulated that further damage may be inflicted during the sharper oscillation phase with more abrupt changes in direction as a result of a lightning strike which may further weaken and break fibers both at the impact point and in areas nearby. In the case of composites with surface mesh, it could also adversely affect the electrical continuity of the mesh and its shielding effectiveness for underlying electric and electronic components. These observations require careful consideration when modelling and designing composite materials and components for applications such as aerospace, with a focus on validating results against experimental work.

\section{Spectral Analysis For Lightning Damage DIAGNOSTICS}

More advanced techniques can be used to analyze the physics of lightning and its interactions with materials to develop fundamental understanding further and validate key assumptions for modelling work. Besides improved methods to measure current, voltage, temperature and pressure gauges, a passive spectrographic system has recently been developed at MBLL capable of identifying chemical element interactions, event temperatures and energy measurements alongside a complimentary transient light system [11-13]. Light from a lightning test event is transported via optic fiber to an optic chassis which spreads the light across its wavelength to produce a spectrum which is subsequently recorded by a digital camera. Distinctive spectral lines, which result from excited states of chemical elements within the lightning arc and any material the arc is impacting, can then be identified in the measured spectrum. Furthermore, using Blackbody Radiation fits, the temperature and energy of the event can then be estimated.

Spectral analysis is of particular significance as it has recently been used to show that, despite a number of differences, the underlying physics of natural and generated lightning strikes are very similar resulting in virtually the same emission spectrum in each case [13]. Figure 5 depicts the spectra with the distinctive chemical element lines clearly visible in both natural lightning, shown in Figure 5(a), and laboratory-generated lightning arcs, shown in Figure 5(b). It has also shown that the temperature of laboratory-generated lightning arcs is similar to that of equivalent natural generated lightning arcs [11-12]. These confirmations expected to lead to verification and validation of material modelling and design work being carried out in lightning laboratories with confidence. That is that laboratory observation and testing with lightning currents is a valid representation of natural lightning events which an aircraft may experience.

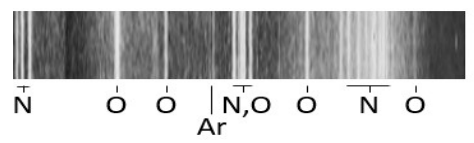

(a) Natural lightning

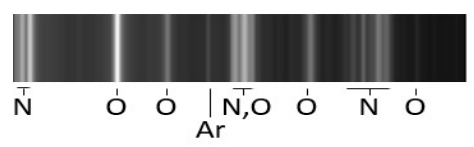

(b) Lightning generated at MBLL

Fig. 5: A section of spectrographic data showing the identification of distinctive chemical element lines.

\section{CONCLUSIONS}

The emerging area of modern lightning research is an increasingly important area of consideration in a wide range of applications, with a current major focus on the use of new carbon composite materials within the aerospace industry. However, this is not just limited to the aerospace industry as 
the introduction of non-conductive composites into other industry sectors, such as wind turbine structures, power infrastructure, shipping and rail electrification, will present similar challenges.

This paper had demonstrated that new measurement techniques help to understand better the interaction of lightning with aircraft materials and to clarify the degradation mechanisms under direct effects of lightning currents. The overall ability to model, predict and design new materials and components to withstand such extreme lightning impact conditions is critical in order to retain their structural integrity and protect electrical and electronic devices. This paper has shown that experimental techniques can support modelling and validate assumptions. Modern lightning generators and associated diagnostic techniques can offer an invaluable resource to achieve this as exemplified with the developed spectrograph system.

\section{ACKNOWLEDGMENTS}

The authors gratefully acknowledge the financial support provided by the Sêr Cymru National Research Network in Advanced Engineering and Materials (NRN073) and Innovate UK via the Aerospace Technology Institute (113037).

\section{REFERENCES}

[1] V. Pasko, Y. Yair and C-L Kuo, "Lightning related transient luminous events at high altitude in the Earth's atmosphere: Phenomenology, mechnisms and effects"; Space Sci. Rev., vol. 168, iss. 1, pp 475-516, June 2012.
[2] N. Reeve and R. Toumi, "Lightning activity as an indicator of climate change"; J. Royal Met. Soc., vol. 125, iss. 555, pp 893-903, Apr 1999.

[3] H. Simpson, P. Leichauer, C. Stone and A. Haddad, "Establishment of a lightning test laboratory for direct effects research"; Int. Conf. on Lightning and Static Electricity, pp. 30, Seattle (USA), September 2013

[4] EUROCAE WG-31 and SAE Committee AE4L ED-84 "Aircraft lightning environment and related waveforms"; 1997

[5] B. Lepetit, F. Soulas, S. Guinard, I. Revel and G. Peres, "Analysis of composite panel damages due to a lightning strike"; Int. Con. on Lightning and Static Electricity, Seattle, USA, September 2013.

[6] Y. Hirano, S. Katsumata, Y. Iwahori and A. Todoroki, "Artificial lightning testing on graphite/epoxy composite laminate"; Comp. A, vol. 41, iss. 10, pp.1461-1470, October 2010.

[7] F. Soulas, B. Lepetit, C. Espinosa, F. Lachaud, S. Guinard, Y. Duval and I. Revel, "How to define a mechanical impact equivalent to a lightning strike"; Int. Con. on Lightning and Static Electricity, Toulouse, France, September 2015

[8] N. S. Jamoshid, D. Mitchard, H. Griffiths and A. Haddad, "Modelling the deflection of carbon fibre reinforced polymer materials under lightning strikes"; Int. Con. on Lightning and Static Electricity, Toulouse, France, September 2015.

[9] D. Mitchard, D. Clark, D. Carr and A. Haddad, "Experimental and theoretical evaluation of aluminium deflection due to lightning strikes", int. Conf. on Lightning Protection, Estoril, Portugal, September 2016

[10] D. Clark, D. Mitchard, C. Stone and A. Haddad, "Spectroscopic characteristics of high-current arcs for a lightning direct-effects laboratory”; Int. Sym. High Voltage, Pilsen, Czech Republic, August 2015

[11] D. Mitchard, D. Clark, D. Stone and A. Haddad, "Preliminary results for spectrographic lightning arc measurements"; Int. Conf. on Lightning and Static Elecricity, Toulouse (France), September 2015

[12] D. Mitchard, D. Clark, D. Carr and A. Haddad, "Technique for the comparison of light spectra from natural and laboratory generated lightning current arcs"; App. Phys. Lett., unpublished at time of print. 